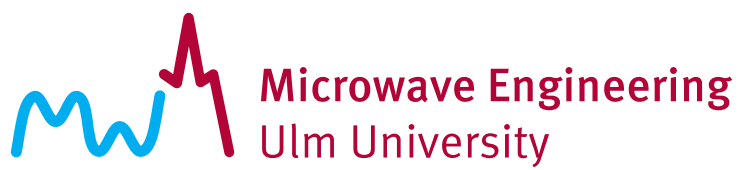

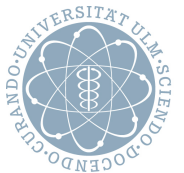

\section{Design of a Dual-Polarized Stacked Patch Antenna for Wide-Angle Scanning Reflectarrays}

T. Chaloun and V. Ziegler and W. Menzel

(C) 2016 IEEE. Personal use of this material is permitted. Permission from IEEE must be obtained for all other uses, in any current or future media, including reprinting/republishing this material for advertising or promotional purposes, creating new collective works, for resale or redistribution to servers or lists, or reuse of any copyrighted component of this work in other works. 


\title{
Design of a Dual-Polarized Stacked Patch Antenna for Wide Angle Scanning Reflectarrays
}

\author{
Tobias Chaloun, Student Member, IEEE, Volker Ziegler, Senior Member, IEEE, \\ and Wolfgang Menzel, Fellow, IEEE
}

\begin{abstract}
A novel dual-polarized stacked patch antenna element for wide-angle scanning Satcom on the move applications at Ka band is presented. The proposed highly integrated multilayer unit cell element operates from $27.8 \mathrm{GHz}$ to $30.8 \mathrm{GHz}$ with excellent scan performance up to $\pm 60^{\circ}$ in both $\mathrm{E}$ - and $\mathrm{H}$-plane. High port-to-port isolation in the entire scan volume evidences the antenna's distinguished suitability for integration into an active folded reflectarray transceiver architecture. Parasitic effects in planar array antennas degrading the intended scan volume are investigated using the infinite array analysis, and measures are introduced to suppress them efficiently. To validate the proposed antenna design several configurations were fabricated and measured successfully. The experimental results show close agreement with the simulations and indicate its excellent scanning capabilities.
\end{abstract}

Index Terms-dual-polarized stacked patch, phased array, reflectarray, scan blindness, surface wave, leaky wave, satcom

\section{INTRODUCTION}

$\mathbf{T}$ HE demand for broadband mobile satellite communication links on platforms like airplanes, trains or ships is growing for a large variety of end users [1], [2]. In mobile communication scenarios the antenna system is typically exposed to a highly dynamic environment. As a consequence, low-profile and lightweight architectures are advantageous to minimize the mechanical impact. This comes along with future satellite communication platforms tending to higher operational frequencies to meet both higher data rates and more compact antenna systems. In order to keep established communication links while moving and to avoid bulky mechanical configurations, planar concepts of electronically steerable antenna systems are of major importance [3], [4].

The proposed architecture for the active transceiver array at $\mathrm{Ka}$ band is based on the folded reflectarray principle [5]. The required operational bandwidth is from $29.5 \mathrm{GHz}$ to $30.8 \mathrm{GHz}$ with beam steering capabilities of $\pm 60^{\circ}$. The general cross section of the array configuration is depicted in Fig. 1 [6].

Considering the array in transmit mode, the primary feed antenna in the center of the array radiates an electromagnetic

Manuscript received August 04, 2015; revised March 17, 2016; accepted April 26, 2016. Date of publication Month xx, 2016; date of current version April 29, 2016. This work was supported by the European Commission's Seventh Framework Programme under Grant agreement number 257335.

T. Chaloun, and W. Menzel are with the Institute of Microwave Engineering, Ulm University, 89081 Ulm, Germany (e-mail: tobias.chaloun@uni-ulm.de). V. Ziegler is with AIRBUS Group Innovations, 81663 Munich, Germany. Color versions of one or more of the figures in this paper are available online at http://ieeexplore.ieee.org.

Digital Object Identifier xx.xxxx/TAP.2016.xxxxxxx

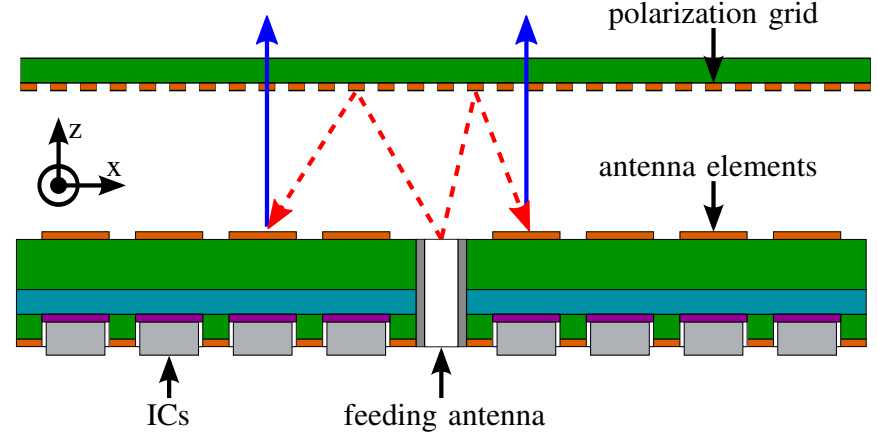

Fig. 1. Principle cross section of the active folded reflectarray transceiver.

wave against the polarizer (dashed ray). Its polarization is aligned with respect to the polarization grid such that the incident wave is reflected back and illuminates the active array. The signal is received by the individual antenna elements and guided to the ICs on the backside of the multilayer PCB. In the active circuits the signal is amplified and adjusted in amplitude and phase according to the required radiation diagram. Subsequently, the modified signal is re-fed to the same antenna elements and re-emitted in the orthogonal polarization, such that the outgoing wave now passes the polarization grid (solid ray). As a result of its operation principle the folded reflectarray is single linear polarized.

In the presented array concept the signal is distributed to the antenna elements by a space-fed arrangement. In comparison to conventional planar arrays [3], [7], [8] the integration of a printed RF distribution network is avoided and the efficiency increases, in particular for larger arrays. Further, the system complexity is reduced, leading to a more flexible and scalable design [9]. In contrast, a more sophisticated antenna design is necessary. The unit cell element requires dual-polarized operation to rotate the polarization of the re-radiated signal by $90^{\circ}$. A high isolation between the two ports is therefore needed since this limits the maximum usable gain of the ICs. As a matter of course, this decoupling has to be maintained for any beam position within the intended scan volume.

Recent investigations on dual-polarized tapered slot array antennas have presented broadband characteristics within a scan volume up to $\pm 45^{\circ}$ from boresight over more than one octave bandwidth [10], [11]. But a significant drawback of this class of antennas is their relatively complex integration into the system. Also the height of tapered slot antennas is inherently large which conflicts with the necessity of a low profile array design. Microstrip patch antennas can meet some of the system 
requirements being lightweight, low profile, and compact due to simple incorporation into the multilayer PCB technology. Unfortunately, conventional microstrip antennas are able to cover only a narrow impedance bandwidth as their electrical volume is small. Using a thicker dielectric material increases the operational bandwidth of the microstrip antenna arrays but in return the usable scan volume decreases since leaky and surface wave resonance effects appear closer to boresight [12], [13]. Several techniques including the use of shorting posts [14] or cavity backing [15]-[17] have been proposed to overcome scan limitations for thicker dielectric materials. Moreover, single polarized aperture stacked patch antennas have been investigated to further enhance the array's scan and bandwidth performance [18]. Thereby scanning capabilities up to $\pm 45^{\circ}$ and $\pm 55^{\circ}$ are obtained for a unit cell size of about $0.437 \lambda_{\min }$ in the E- and H-plane, respectively.

As opposed to the entire system design [6] in this paper the dual-polarized aperture coupled stacked patch antenna is investigated in detail for the use in large folded reflectarray antennas. It will be shown that the novel unit cell design is able to operate within a scanning range of $\pm 60^{\circ}$ in the principal planes. To the authors' knowledge, this is the largest scan volume yet achieved for a dual-polarized patch antenna array considering a unit cell size of $0.513 \lambda_{\min }$. In-depth design considerations and the analysis of the port-to-port isolation as a function of the scan angle is presented which is unique in literature.

The paper is organized as follows. Section II describes the unit cell configuration and design strategies to shift parasitic effects out of the intended scan volume. Experimental results of realized the antenna prototypes are discussed in Section III. Finally, the conclusion is given in Section IV.

\section{UNIT CELL DESIGN}

As mentioned before the folded reflectarray transceiver operates in the frequency band from $29.5 \mathrm{GHz}$ to $30.8 \mathrm{GHz}$. The electronic beam steering is intended to cover a scan volume up to $60^{\circ}$ in elevation for all planes. In order to achieve a good antenna performance even for larger scan angles, the element size needs to be reasonable small [19]. On the other hand, the minimum dimensions for the unit cell lattice are likewise determined by the achievable integration level. In the presented work the unit cell spacing is set to $l_{\mathrm{uc}}=5 \mathrm{~mm}$ using a non-skewed square lattice. This corresponds to the onset of higher order Floquet modes, which manifest in grating lobes at

$$
f_{\mathrm{GL}}=\frac{c_{0}}{l_{\mathrm{uc}}\left|1+\sin \left(\vartheta_{\max }\right)\right|}=32.15 \mathrm{GHz},
$$

where $\vartheta_{\max }=60^{\circ}$.

However, scan blindness in planar phased arrays may occur closer to boresight caused by leaky and surface wave resonances [19]-[21]. Novel multilayer antenna concepts are necessary to meet all objectives within the limited lateral extent. In Fig. 2 the exploded view of the proposed dualpolarized aperture coupled stacked patch antenna for wide scanning applications is shown. The novel hybrid antenna configuration is composed of a multilayer PCB, a metal grid, and a thin RF dielectric sheet on the very top. The

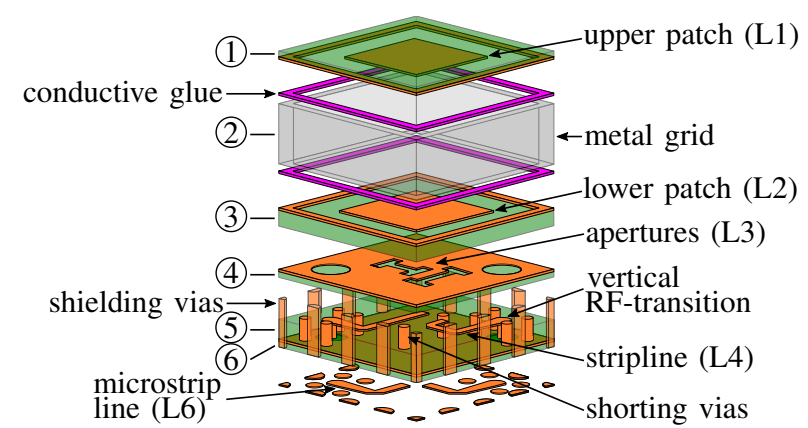

Fig. 2. Exploded view of the dual-polarized aperture coupled stacked patch antenna.

multilayer PCB contains the lower patch and two apertures fed by stripline for both polarizations. The vertical transition realized as quasi-coaxial line [22] connects the stripline to the respective microstrip line on the bottom side. Vias surrounding the unit cell are employed to improve the shielding between the adjacent elements and suppress surface wave modes. The RF laminate on top acts as a carrier for the upper patch. At the same time, this serves as a protective layer for the array. The metal grid in between both patches assures a certain distance to adjust the coupled resonances. In addition, mutual coupling is reduced, and the large air volume below the upper patch contributes to an increased operational bandwidth.

A parametric study and optimization of the proposed array antenna have been carried out using Ansys HFSS [23]. To obtain its performance under different scan angles, the antenna element is considered as a unit cell in an infinite periodic environment. The entire unit cell design of the dual-polarized stacked patch antenna offers many degrees of freedom as can be seen from Fig. 3. Therefore, the structure for the vertical

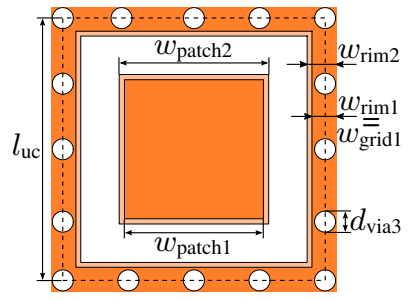

(a) L1, L2

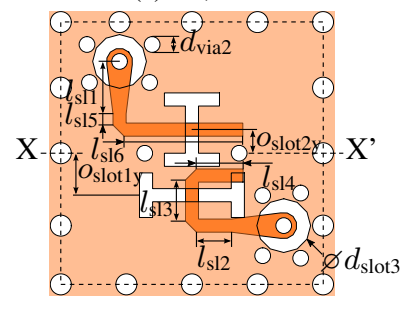

(c) L3, L4

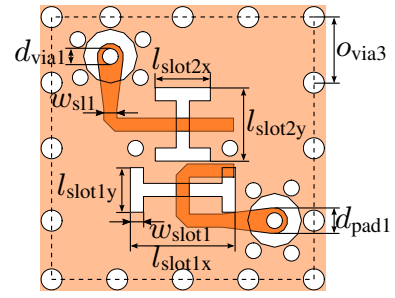

(b) L3, L4

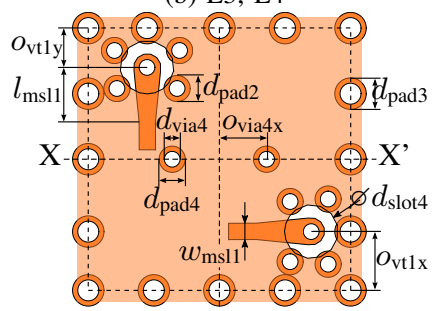

(d) L5, L6
Fig. 3. Parameters of the dual-polarized aperture coupled stacked patch antenna (dimensions of both ports are identical unless otherwise specified).

transition was optimized separately to reduce the complexity of the problem. Besides, the substrate layers (4), (5), (6) (see Fig. 2), which have minor impact on the radiation properties, were fixed in this step, too. 
Dual-polarized antenna designs using superposed apertures, for instance a centered cross-shaped slot, may achieve excellent isolation levels due to the high degree of symmetry. But the excitation requires a relatively large balanced feed network for each polarization to maintain this symmetry and the resultant high polarization decoupling [24]. As a consequence of the discussed area constraints within the unit cell element, an offset $\mathrm{H}$-shape aperture for each polarization is applied, since its excitation by a compact single stripline feed results only in a slightly degradation of the port-to-port isolation.

Moreover, shorting vias between both ground planes were introduced since the aperture fed by stripline causes parasitic coupling to parallel-plate modes [25], [26]. The height of the dielectric (3) was chosen such that the coupling between the aperture and the lower patch is adjusted similarly for both polarizations. The thickness of the superstrate (1) is kept as thin as possible to move the excitation of leaky and surface wave resonances to larger scan angles. A more in-depth discussion will be presented later. Finally, both coupled resonances of the aperture stacked patch antenna were tuned by the dimensions of the square patches and the metal grid height (2) for appropriate impedance matching in the operational frequency band [27]. The dimensions of the novel stacked patch antenna design depicted in Fig. 3 are listed in Table I. The material composition of the final design is given in Table II.

TABLE I

DIMENSIONS OF THE APERTURE COUPLED STACKED PATCH ANTENNA.

\begin{tabular}{|c|c|c|c|c|c|}
\hline$l_{\mathrm{uc}}$ & $5.00 \mathrm{~mm}$ & $w_{\text {patch1 }}$ & $2.65 \mathrm{~mm}$ & $w_{\text {patch2 }}$ & $2.85 \mathrm{~mm}$ \\
\hline$\overline{w_{\text {rim1 }}}$ & $0.25 \mathrm{~mm}$ & $w_{\text {rim2 }}$ & $0.30 \mathrm{~mm}$ & $w_{\text {slot } 1}$ & $0.25 \mathrm{~mm}$ \\
\hline$l_{\text {slot } 1 \mathrm{x}}$ & $2.00 \mathrm{~mm}$ & $l_{\text {slot1y }}$ & $0.85 \mathrm{~mm}$ & $O_{\text {slot1y }}$ & $0.80 \mathrm{~mm}$ \\
\hline$l_{\text {slot } 2 \mathrm{x}}$ & $1.05 \mathrm{~mm}$ & $l_{\text {slot2y }}$ & $1.40 \mathrm{~mm}$ & $O_{\text {slot2y }}$ & $0.45 \mathrm{~mm}$ \\
\hline$d_{\text {slot } 3}$ & $1.03 \mathrm{~mm}$ & $d_{\text {slot } 4}$ & $1.03 \mathrm{~mm}$ & $O_{\mathrm{vt} 1 \mathrm{x}}$ & $1.12 \mathrm{~mm}$ \\
\hline$O_{\mathrm{vt} 1 \mathrm{y}}$ & $0.75 \mathrm{~mm}$ & $w_{\mathrm{s} 11}$ & $0.25 \mathrm{~mm}$ & $w_{\mathrm{msl} 1}$ & $0.30 \mathrm{~mm}$ \\
\hline$l_{\mathrm{s} 11}$ & $1.00 \mathrm{~mm}$ & $l_{\mathrm{s} 2}$ & $0.63 \mathrm{~mm}$ & $l_{\mathrm{s} 13}$ & $0.71 \mathrm{~mm}$ \\
\hline$l_{\mathrm{s} 14}$ & $0.85 \mathrm{~mm}$ & $l_{\mathrm{s} 15}$ & $0.18 \mathrm{~mm}$ & $l_{\mathrm{s} 16}$ & $2.23 \mathrm{~mm}$ \\
\hline$d_{\text {pad1 }}$ & $0.50 \mathrm{~mm}$ & $d_{\text {pad2 }}$ & $0.50 \mathrm{~mm}$ & $d_{\text {pad3 }}$ & $0.60 \mathrm{~mm}$ \\
\hline$d_{\mathrm{pad} 4}$ & $0.50 \mathrm{~mm}$ & $d_{\text {via1 }}$ & $0.30 \mathrm{~mm}$ & $d_{\mathrm{via} 2}$ & $0.30 \mathrm{~mm}$ \\
\hline$d_{\text {via3 }}$ & $0.40 \mathrm{~mm}$ & $d_{\text {via } 4}$ & $0.30 \mathrm{~mm}$ & $o_{\text {via3 }}$ & $1.25 \mathrm{~mm}$ \\
\hline$O_{\text {via } 4 x}$ & $0.90 \mathrm{~mm}$ & & & & \\
\hline
\end{tabular}

TABLE II

DIELECTRIC MATERIALS OF THE PROPOSED STACKED PATCH ANTENNA.

\begin{tabular}{|c|c|c|c|}
\hline No. & Material & Height / mm & Rel. permittivity \\
\hline \hline (1) & Rogers RT5880 & 0.127 & 2.2 \\
\hline (2) & Air & 1.65 & 1.0 \\
\hline (3) & Rogers RT5880 & 0.381 & 2.2 \\
\hline (4) & Rogers RO3003 & 0.127 & 3.0 \\
\hline (5) & Rogers RO3003 & 0.508 & 3.0 \\
\hline (6) & Rogers RO3003 & 0.127 & 3.0 \\
\hline
\end{tabular}

The threshold for an acceptable reflection coefficient within the intended scan volume is taken to be $-10 \mathrm{~dB}$. The computed reflection coefficients of the proposed dual-polarized aperture stacked patch antenna are shown in Figs. 4 and 5 for scanning fin the E- and H-plane, respectively. In the frequency range from $27.8 \mathrm{GHz}$ to $30.8 \mathrm{GHz}$ the reflection coefficient remains below $-10 \mathrm{~dB}$ even for scan angles up to $60^{\circ}$ in both principle planes. Due to the asymmetrical arrangement of the two

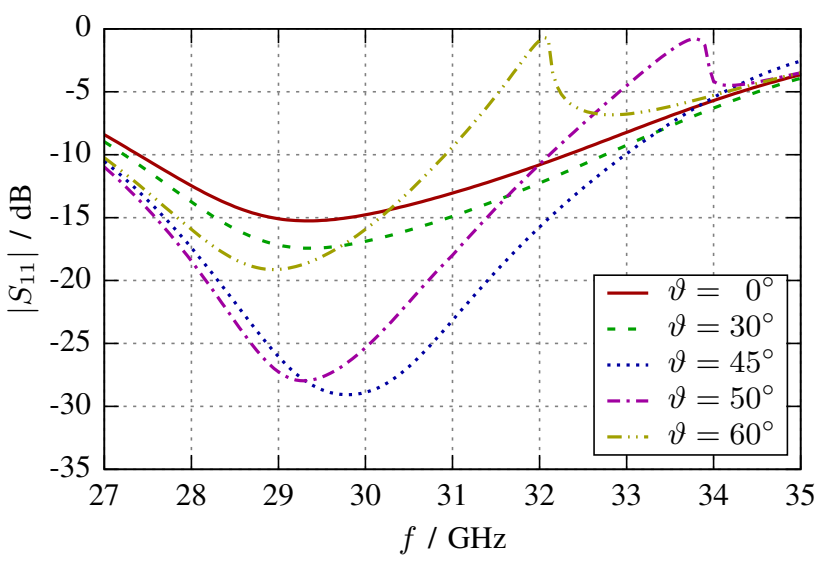

(a) Port 1

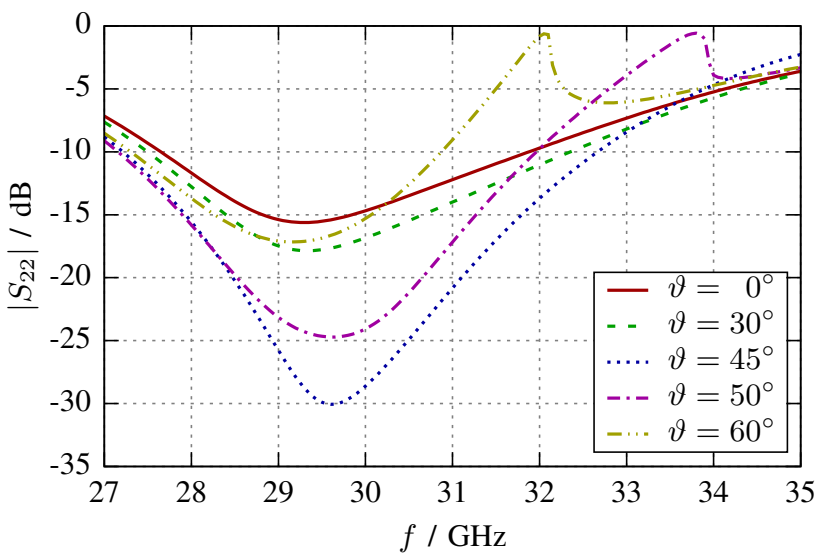

(b) Port 2

Fig. 4. Simulated reflection coefficients at different scan angles in the E-plane.

feeding structures their performance is not identical. During the design process efforts were made to compensate this issue. As a result the ports of the unit cell show very similar behavior. For E-plane scanning a rapid rise of the reflection coefficient is observed close to the onset of the free space grating lobe. The grating lobe in the E-plane corresponds to the $\operatorname{TM}(-1,0)$ Floquet mode and causes a singularity in the admittance plane when it enters into the visible region at $f_{\mathrm{GL}}=32.15 \mathrm{GHz}$ for $60^{\circ}$ off-boresight. At this points the reflection coefficient approaches $0 \mathrm{~dB}$ resulting in scan blindness. In contrast, the $\mathrm{H}$-plane scan performance at $60^{\circ}$ exhibits a less pronounced discontinuity due to the absence of singularities. Here the small dip at $32.15 \mathrm{GHz}$ may be associated with strong mutual coupling between the TM and TE Floquet modes [12], [26].

The corresponding port-to-port coupling coefficient (see Fig. 6) illustrates this effect from a different perspective. Across the frequency range from $27.5 \mathrm{GHz}$ to $31.5 \mathrm{GHz}$ the coupling level between both feedings is always below $-25 \mathrm{~dB}$ and has its maximum at the cut-off frequency for the $\operatorname{TM}(-1,0)$ and $\operatorname{TE}(0,-1)$ Floquet mode, respectively.

In spite of this, the usable scan volume in planar phased arrays is mainly limited by leaky and surface wave resonances as they arise before the onset of the grating lobe. Degradation in the scan performance or even scan blindness can occur due to forced guided wave resonances on either antenna or 


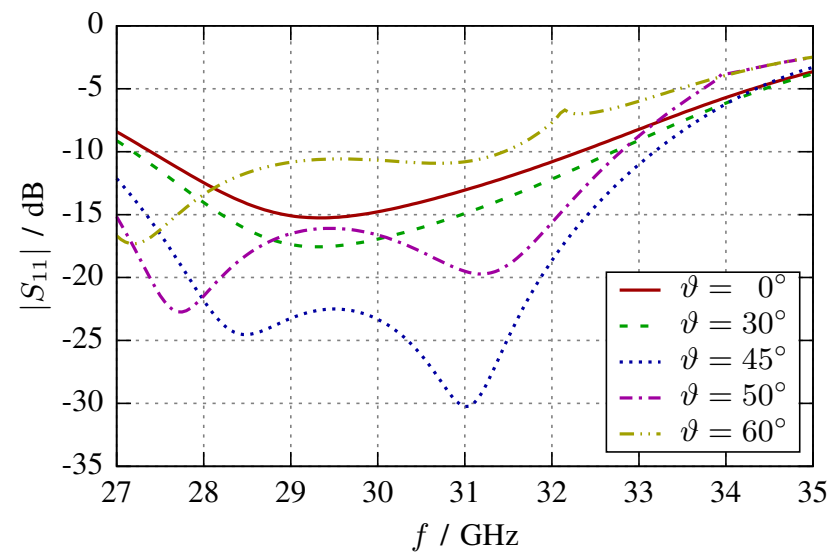

(a) Port 1

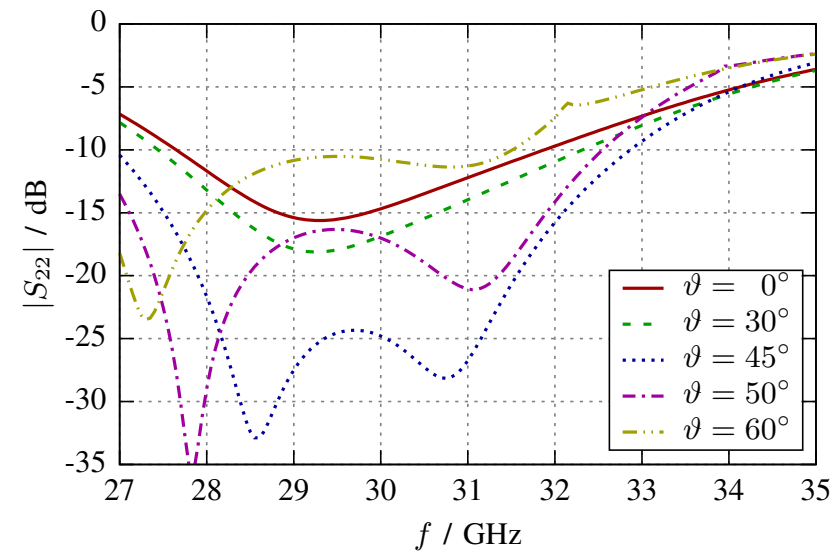

(b) Port 2

Fig. 5. Simulated reflection coefficients at different scan angles in the $\mathrm{H}-$ plane.

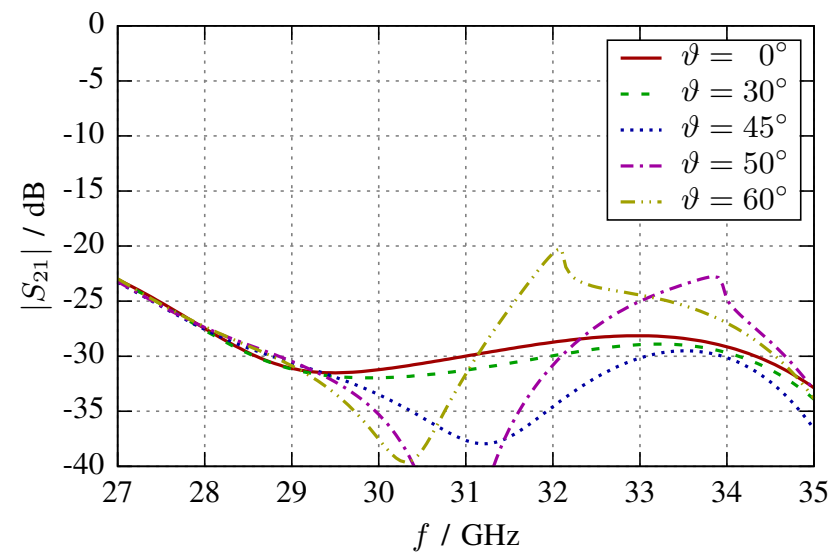

Fig. 6. Simulated port-to-port coupling coefficient $\left|S_{21}\right|$ at different scan angles.

feed substrates [13]. In the proposed antenna configuration the superstrate (1) represents the dominant parameter to reduce those parasitic effects. In the following discussion the impedance of the $\operatorname{TM}(0,0)$ Floquet mode is defined at the aperture plane as

$$
Z_{00}^{\mathrm{FTM}}=R_{00}^{\mathrm{FTM}}+\mathrm{j} X_{00}^{\mathrm{FTM}}
$$

where $R_{00}^{\mathrm{FTM}}$ is the radiation resistance and $X_{00}^{\mathrm{F}, \mathrm{TM}}$ the corresponding reactive component.

Fig. 7 shows the effect of the superstrate height on the resistance of the $\operatorname{TM}(0,0)$ Floquet mode for E-plane scanning at $60^{\circ}$. The minimum of the resistance $R_{00}^{\mathrm{F}, \mathrm{TM}}$ is related to the $\mathrm{TM}_{0}$ surface wave on the unloaded dielectric slab. The real part of the dominant TM Floquet mode impedance becomes very low but finite, whenever a phase-match condition between the inclined $\operatorname{TM}(0,0)$ mode and the surface wave resonance is met [28]. The phase constant of the TM surface wave $\beta_{\text {SW }}$ increases with the height of the dielectric material (1) [29]. The elevation angle at which the distinctive scan blindness appears may be calculated by

$$
\sin \left(\vartheta_{\mathrm{SW}}\right)=\left|\frac{\beta_{\mathrm{SW}}}{k_{0}}-\frac{\lambda_{0}}{l_{\mathrm{uc}}}\right| \text {. }
$$

Accordingly, the minimum resistance moves closer to boresight for thicker dielectric slabs.

The maximum of $R_{00}^{\mathrm{F}, \mathrm{TM}}$ is associated with a leaky wave guided on the dielectric slab loaded by both the upper patch and the metal grid [21]. As seen from Fig. 7, the phase-match condition between the leaky wave resonance and the dominant Floquet mode occurs at lower frequencies than for the surface wave resonance. The reason is found in the fast wave nature of leaky wave modes [30]. The peak in the resistance moves closer to boresight for thicker superstrates and manifests in a strong mismatch but not in complete array blindness. Furthermore, the thinner the superstrate the higher the quality factor $Q_{\mathrm{LW}}$ of the leaky wave resonance. Since the dielectric for the upper patch with $\epsilon_{r}=2.2$ and $h_{\text {diel } 1}=0.127 \mathrm{~mm}$ is chosen to be electrically thin, neither surface nor leaky wave resonances occur for the H-plane scan.

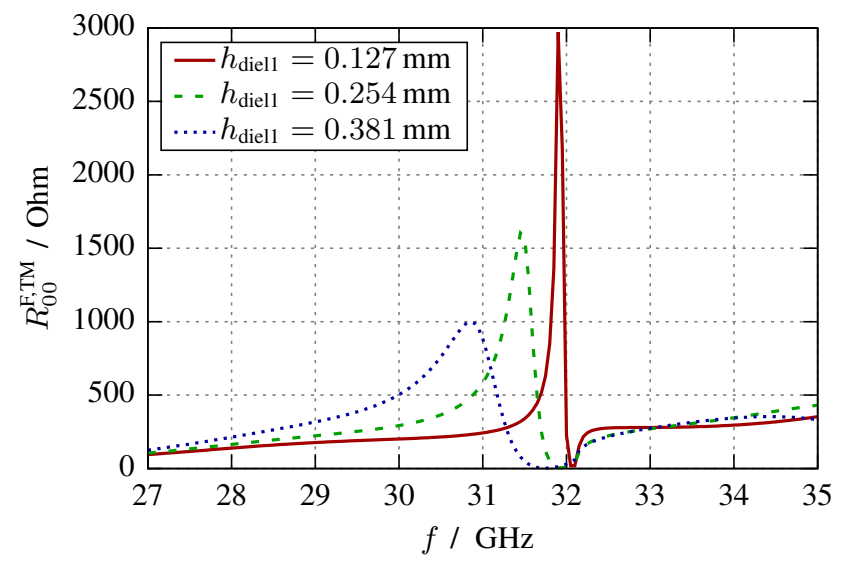

Fig. 7. Simulated resistance of the $\operatorname{TM}(0,0)$ Floquet mode for E-plane scanning at $60^{\circ}$.

Figs. 8 and 9 depict the simulated transmission coefficients from port 1 to the fundamental Floquet mode for scanning in the E- and H-plane, respectively. In some way, the simulated reflection coefficients indicate the array's scan performance, but the introduced transmission coefficients according to Figs. 8 and 9 offer a more precise evaluation criterion in terms of the radiation efficiency [20]. On condition that the dual linear polarized array antenna is placed in an infinite 


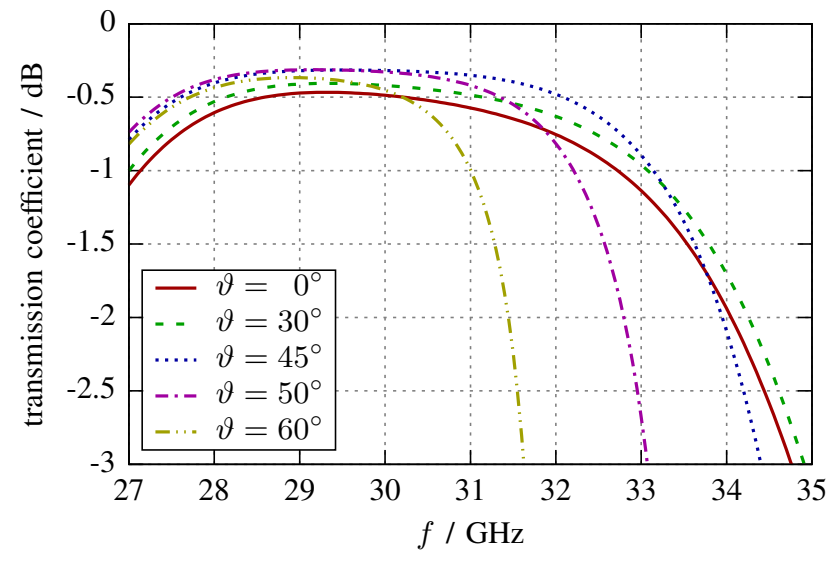

(a) Co-polarization

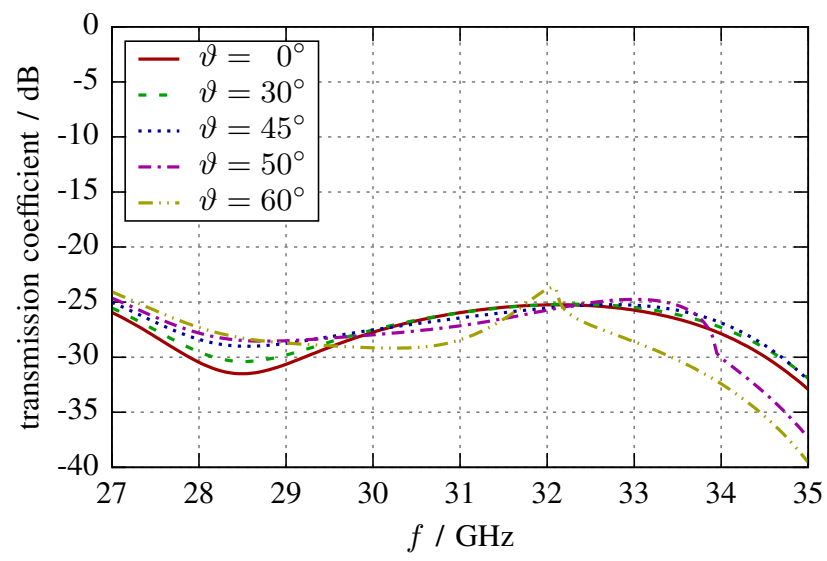

(b) Cross-polarization

Fig. 8. Simulated transmission coefficients for scanning in the E-plane (port 1).

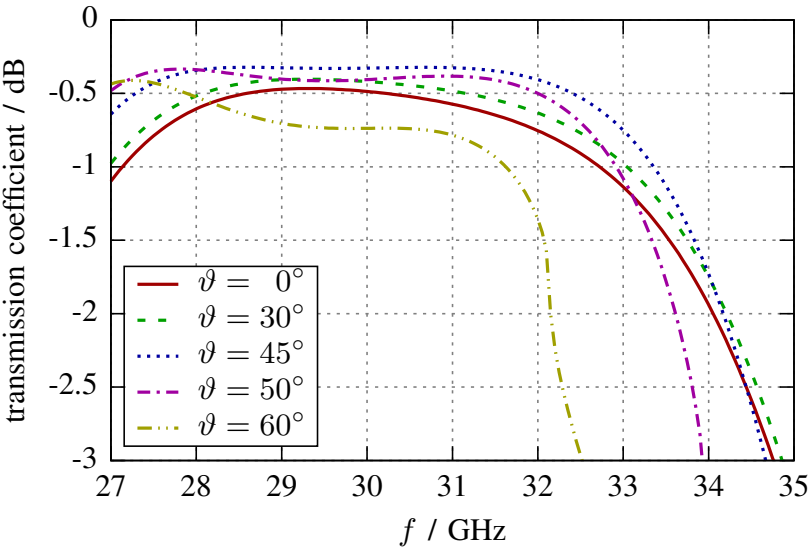

(a) Co-polarization

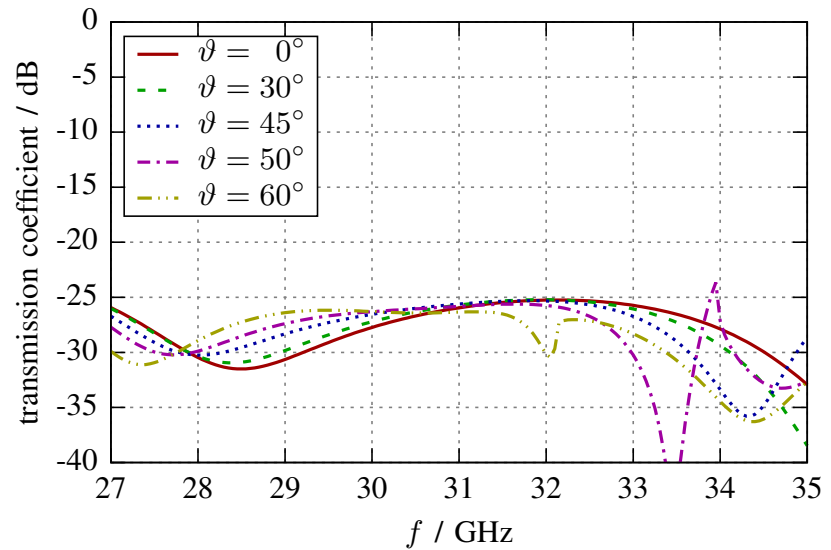

(b) Cross-polarization

Fig. 9. Simulated transmission coefficients for scanning in the H-plane (port 1).

periodic environment, the co-polarization of the transmission coefficient describes the coupling to the designated $\operatorname{TM}(0,0)$ and $\operatorname{TE}(0,0)$ Floquet mode for the E- and H-plane scan, respectively. Consequently, the cross-polarization level shows the coupling to the fundamental Floquet mode perpendicular to the designated one. Due to the very similar properties of both ports, only the simulated transmission coefficients with respect to port 1 are presented here. In the E-plane the transmission coefficient from port 1 to the $\operatorname{TM}(0,0)$ Floquet mode (see Fig. 8a) is better than $-0.8 \mathrm{~dB}$ in the whole operational band $(29.5 \mathrm{GHz}-30.8 \mathrm{GHz})$ up to scan angles of $60^{\circ}$. The corresponding coupling to the orthogonal polarization, in this case to the $\operatorname{TE}(0,0)$ Floquet mode, is depicted in Fig. $8 \mathrm{~b}$ and remains always below $-27 \mathrm{~dB}$. The scan performance in the H-plane (see Fig. 9a) shows a spectrally broader transmission to the co-polarization at $60^{\circ}$ than for the E-plane scan. This difference may be consistently explained by reflection losses due to the aforementioned leaky wave resonance in the vicinity of the upper frequency for E-plane scanning. However, the supported TM guided waves would not be excited in the Hplane because of the inherent polarization mismatch.

\section{EXPERIMENTAL RESULTS}

The novel dual-polarized stacked patch antenna fed by microstrip lines was fabricated in several configurations. For the single and double element prototype the metal grid in between the hybrid configuration has been manufactured completely from aluminum using a standard milling process. In a different way, the metal grid for the $11 \times 11$ array prototype has been replaced by a metal-plated FR4 grid structure. The thickness of the galvanized copper cladding and gold surface finish is about25@cmdum. Compared with solid metal grids, the difference in the electrical conductivity due to the skin effect can definitely be neglected here. The obtained accuracy of the standard as well as the low-cost manufacturing technique are both within a tolerance of $\pm 5 @ \mathrm{cmd \mu m}$. The individual parts as shown in Fig. 2 are joined together using a conductive glue. The achieved planarity, accuracy in the alignment, and stability of the assembled hybrid boards show excellent results.

\section{A. Single element prototype}

The measured reflection coefficients of the two ports for the realized single antenna element are presented in Fig. 10. The 
measurement results of the dual-polarized aperture stacked patch antenna show excellent agreement to those of the simulation. In comparison to the unit cell results of the infinite array a slightly larger $10 \mathrm{~dB}$ return loss bandwidth from $26.0 \mathrm{GHz}$ to $32.7 \mathrm{GHz}$ is obtained due to the nonexistence of mutual coupling to adjacent cells. The additional notch in the measurements at $30 \mathrm{GHz}$ is created by a slight translation and rotation of the two resonance loops within the complex reflection plane.

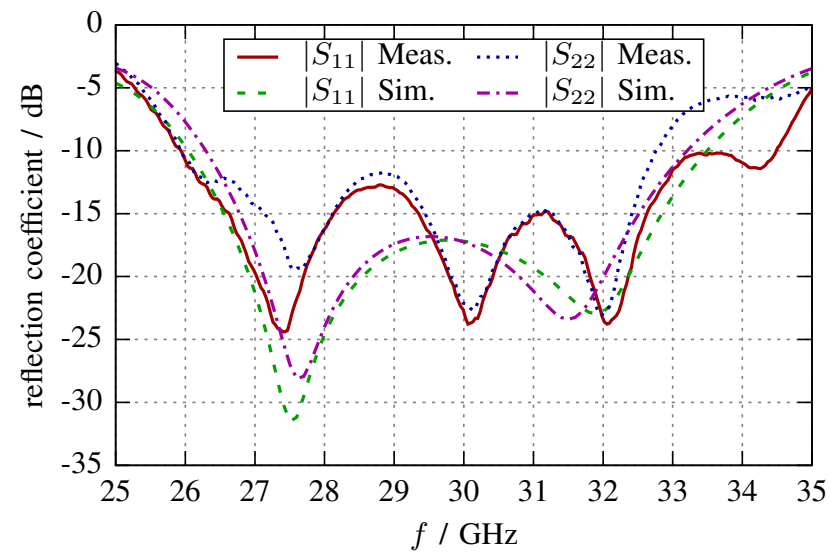

Fig. 10. Measured and simulated reflection coefficients of the single dualpolarized antenna element.

As can be seen in Fig. 11, the measured port-to-port coupling coefficient is better than $-28.5 \mathrm{~dB}$ in the operational band from $29.5 \mathrm{GHz}$ to $30.8 \mathrm{GHz}$ and still below $-20 \mathrm{~dB}$ in the $10 \mathrm{~dB}$ return loss bandwidth. For the potential use in the presented active reflectarray architecture (see Fig. 1) high-gain amplifiers can be introduced into the cross-polarized unit cell without causing instability issues.

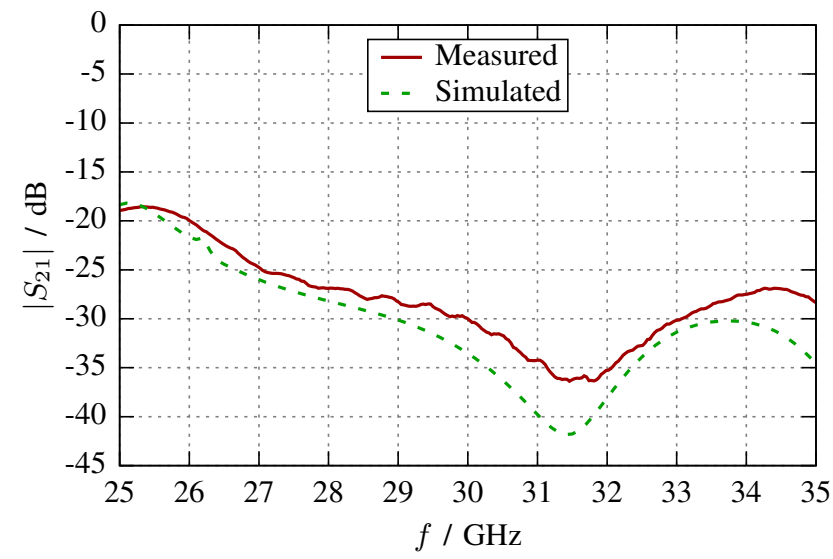

Fig. 11. Measured and simulated port-to-port coupling coefficient $\left|S_{21}\right|$ of the single dual-polarized antenna element.

The radiation characterization of the dual-polarized antenna was conducted for both ports from $26.0 \mathrm{GHz}$ to $32.7 \mathrm{GHz}$. The results at the center frequency $(30 \mathrm{GHz})$ for port 1 are shown in Fig. 12. The measured and simulated results of the co-polarization illustrate an excellent agreement in the E- as well as in the H-plane. The cross-polarization levels remain below $-20 \mathrm{~dB}$. The gain of the single antenna element varies across the measured frequency range between $6.0 @ \mathrm{cmddBi}$ and6.6@cmddBi.

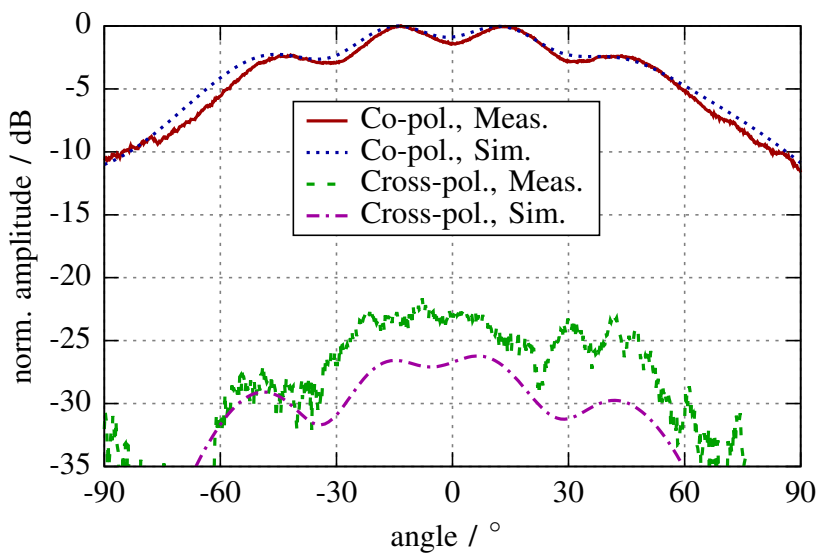

(a) E-plane

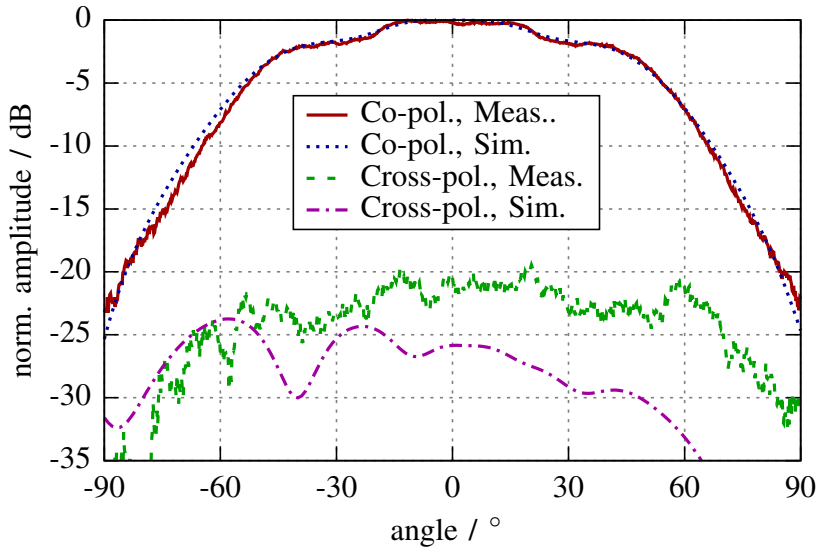

(b) H-plane

Fig. 12. Measured and simulated radiation diagram of the single dualpolarized antenna element at $30 \mathrm{GHz}$ (port 1).

\section{B. Double element prototype}

In order to achieve a high integration level for the active array architecture it is favorable to group the radiating elements into submodules. According to [6] it is assumed that each subarray comprises $2 \times 2$ antenna elements connected to a single multi-functional MMIC. Therefore, the periodicity given by the Floquet analysis of the unit cell must be broken in such a manner so that the active circuit may be placed within the subgroup. As a consequence, the dual-polarized patch antenna is transfered into a finite array environment to assess mutual coupling since the Floquet theory is not reasonably applicable anymore. The considered finite arrays are depicted in Fig. 13. Model I exhibits the same periodicity as used for the Floquet analysis in Section II and serves as reference for the modified Model II. Both configurations consist of $6 \times 6$ dual-polarized antennas in which only the 4 centered elements (gray shaded) are evaluated. The other elements are terminated in matched loads to mimic the field distribution of an infinite array. For the verification of the simulated coupling factors, however, Model II has been reduced to a double element 


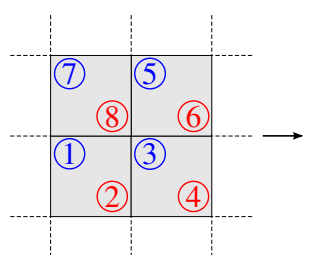

Model I $(6 \times 6)$

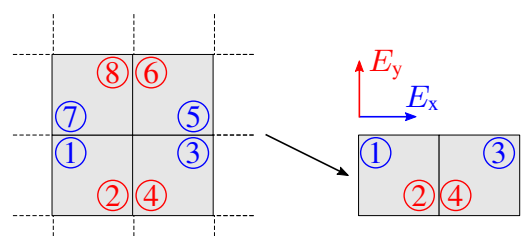

Model II $(6 \times 6)$
Model III $(2 \times 1)$

Fig. 13. Finite array configurations of the dual-polarized stacked patch antenna.

configuration (Model III). The photograph in Fig. 14 shows the realized parts of the two dual linear polarized antenna elements. The arrow next to the port number indicates the polarization of the electromagnetic wave in the far field excited by the corresponding feed.

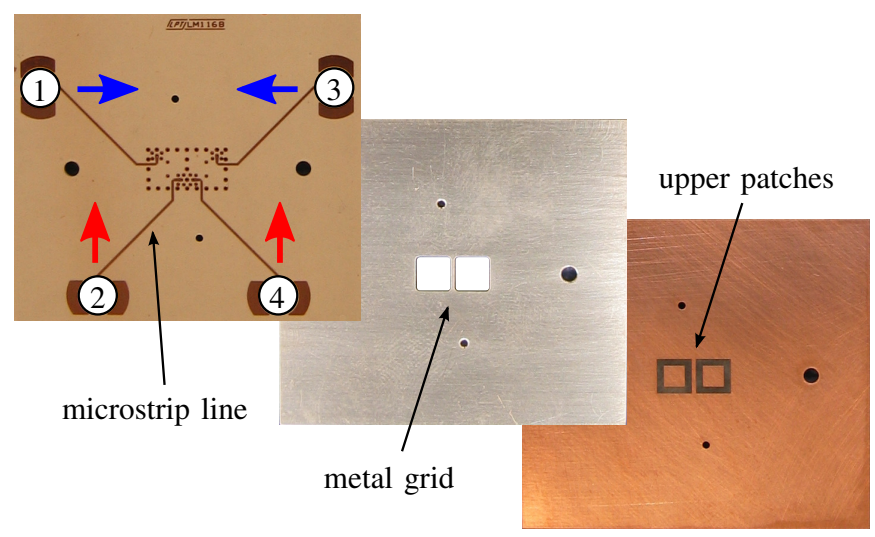

Fig. 14. Photograph of the realized parts for the double element configuration of the proposed dual linear-polarized antenna design (bottom view).

The $10 \mathrm{~dB}$ return loss bandwidth is covered by both dualpolarized antenna elements within a frequency range from $26.1 \mathrm{GHz}$ to $32.9 \mathrm{GHz}$. Owing to the very similar reflection coefficients as for the the single element prototype (see Fig. 10), their illustrations have been omitted.

The mutual coupling between the co-polarized ports for the three different models is exemplarily shown in Fig. 15a. The remaining coupling coefficients have either very similar or higher isolation properties. The comparison of Model I and Model II shows, as expected, that in the latter one $\left|S_{31}\right|$ is lower and in return $\left|S_{42}\right|$ higher. The systematic error made by the simplified Model III with respect to Model II is for the co-polarized coupling coefficients from $29.5 \mathrm{GHz}$ to $30.8 \mathrm{GHz}$ at most $2 \mathrm{~dB}$. The measurement results are slightly lower than predicted from full-wave simulations. In the intended frequency band the measured coupling level shows a very flat response and remains below $-20.5 \mathrm{~dB}$ in the E-plane $\left(S_{42}\right)$ and below $-17.5 \mathrm{~dB}$ in the H-plane $\left(S_{31}\right)$, respectively. Hence, the accepted inaccuracy of this model can be easily de-embedded from the measurements. As mentioned before, parasitic coupling into the orthogonal polarization even from adjacent elements is a crucial factor in active folded reflectarray antennas. Unlike coupling to the same polarization, this effect can not be incorporated into the future pattern synthesis. Fig. 15b depicts the measured and simulated coupling coefficients between orthogonal ports. In accordance with all simulation models, which differ only to a minor degree, the measured cross-coupling component of the neighboring element $\left(\left|S_{41}\right| \leq-42.4 \mathrm{~dB}\right)$ is considerably lower as of the unit cell itself $\left(\left|S_{21}\right| \leq-28.8 \mathrm{~dB}\right)$ in the entire band of interest. As a result, the stability condition of an active unit cell can be approximated by considering self-induced crosscoupling only.

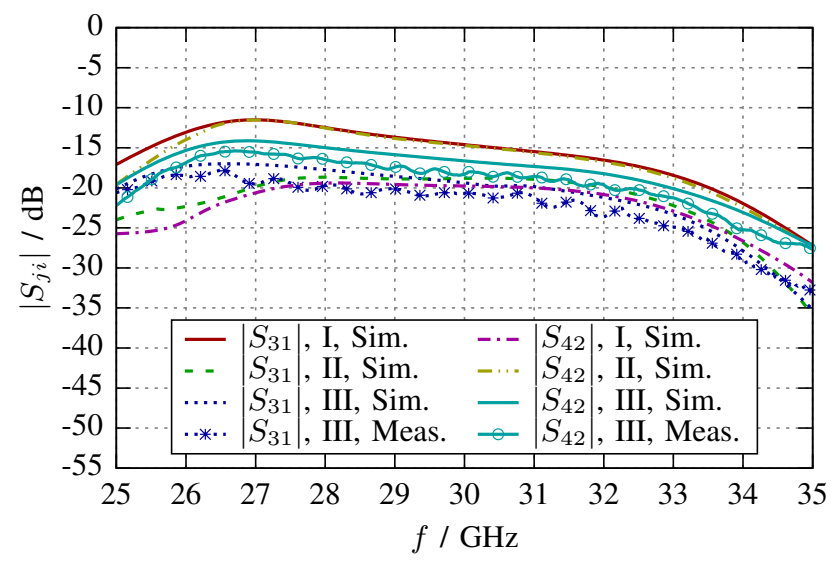

(a) Co-polarization

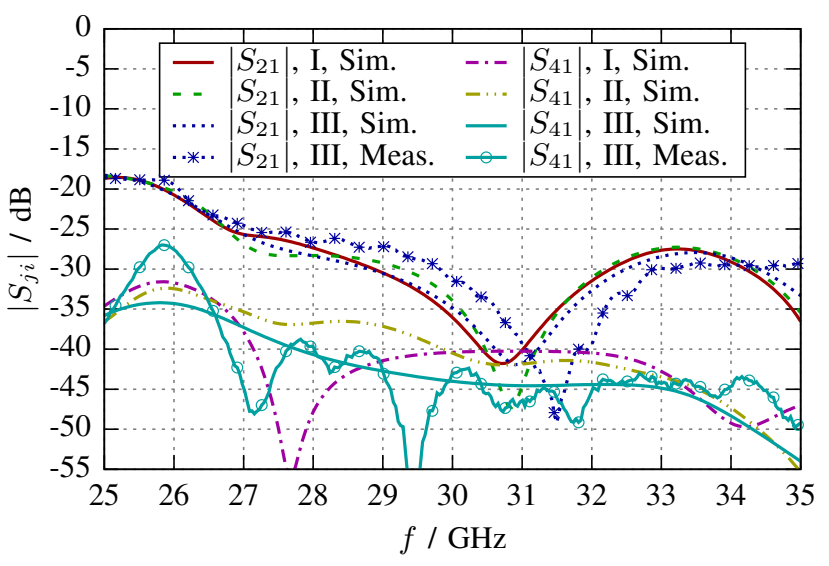

(b) Cross-polarization

Fig. 15. Measured and simulated coupling coefficients of the double element prototype (The roman numbers refer to the appropriate model).

\section{C. $11 \times 11$ array prototype}

Based on the proposed dual-polarized antenna element, also a $11 \times 11$ array prototype has been designed to validate its wide-angle scanning capabilities within an array environment. As this passive array configuration is used to predict the scan performance of a fully excited array by means of the embedded element pattern, only the two ports of the central element are fed. All other ports are terminated with a structure consisting of two $100 \Omega$ resistors in parallel. In this way, all mutual coupling effects are considered and thus visible in the embedded element pattern including those which cause scan blindness [31]. The fabricated and assembled array prototype with $11 \times 11$ dual-polarized stacked patch antennas is illustrated in Fig. 16. The recognizable asymmetry on the bottom side of the multilayer PCB is dictated by the relatively 
large dimensions of the commercial coaxial connector. The compromise made in the design, however, is limited solely to this outer layer.

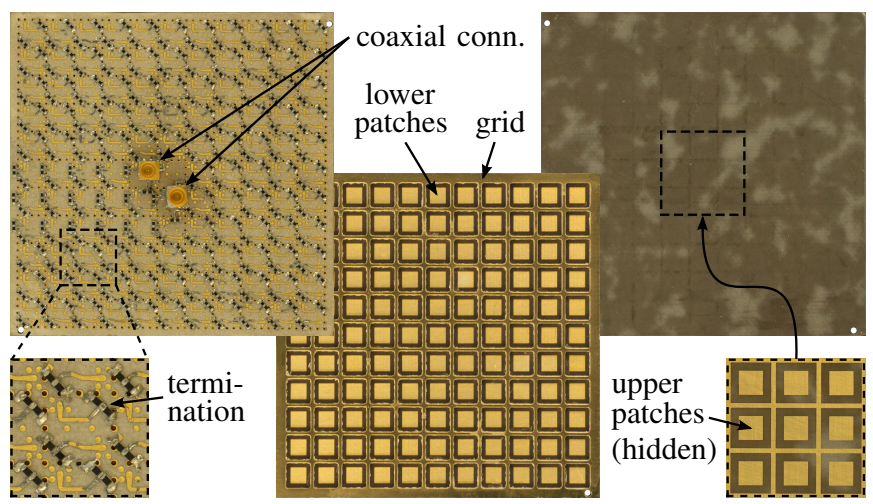

Fig. 16. Photograph of the realized $11 \times 11$ array prototype (bottom, inside, and top view).

The measured reflection coefficients of the embedded dualpolarized stacked patch antenna are depicted in Fig 17. The $10 \mathrm{~dB}$ return loss bandwidth is covered by both ports within the frequency range from $26.5 \mathrm{GHz}$ to $32.7 \mathrm{GHz}$. Besides a slight shift of the coupled resonance between the aperture and lower patch towards lower frequencies, a very good agreement between the measurement and simulation results can be observed. Further it is noteworthy that the reflection coefficients of the embedded and isolated radiating element exhibit very similar behavior (cf. Fig. 10) indicating its robustness against environmental changes.

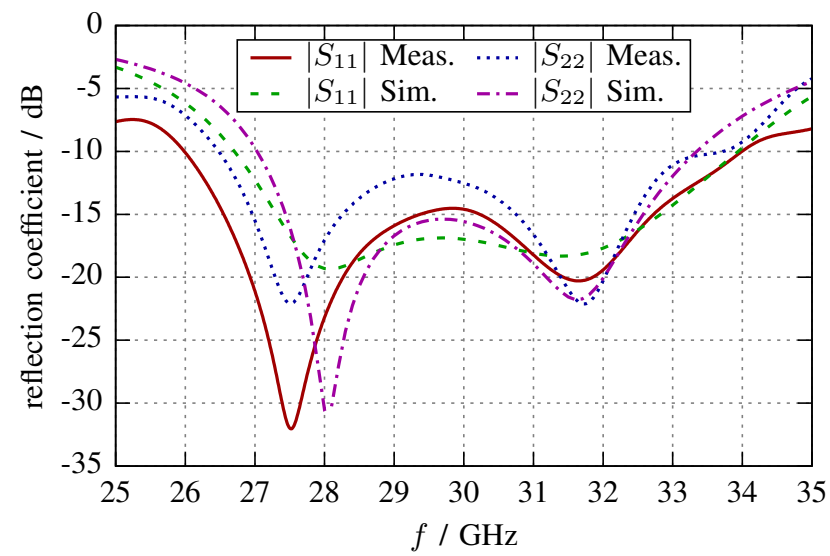

Fig. 17. Measured and simulated reflection coefficients of the embedded dual-polarized stacked patch antenna prototype.

Fig. 18 shows the corresponding port-to-port coupling coefficient of the embedded dual-polarized stacked patch antenna prototype. As can be seen from the measurement results, the coupling level remains well below $-32.5 \mathrm{~dB}$ in the band of interest $(29.5 \mathrm{GHz}-30.8 \mathrm{GHz})$ and below $-24.5 \mathrm{~dB}$ in the $10 \mathrm{~dB}$ return loss bandwidth. The minor deviation from the simulation results may be found in the limited accuracy of the simulation model that has been accepted to overcome the computational effort of the large and complex finite array structure.

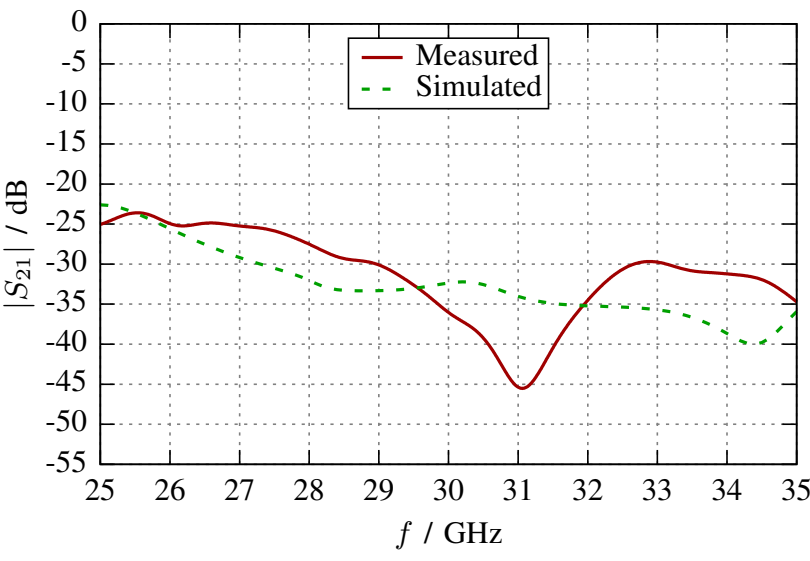

Fig. 18. Measured and simulated port-to-port coupling coefficient $\left|S_{21}\right|$ of the embedded dual-polarized stacked patch antenna prototype.

Taking into account the $10 \mathrm{~dB}$ return loss bandwidth obtained from the infinite array analysis (cf. Section II), the measurements of the radiation characteristics have been conducted for both ports from $28 \mathrm{GHz}$ to $32 \mathrm{GHz}$. The realized gain of the embedded dual-polarized stacked patch antenna is given in Fig. 19. For both polarizations the measured gain is found to be in good agreement with the simulations. The variation is less than $1 \mathrm{~dB}$ and can be attributed to the finite dimensions of the array.

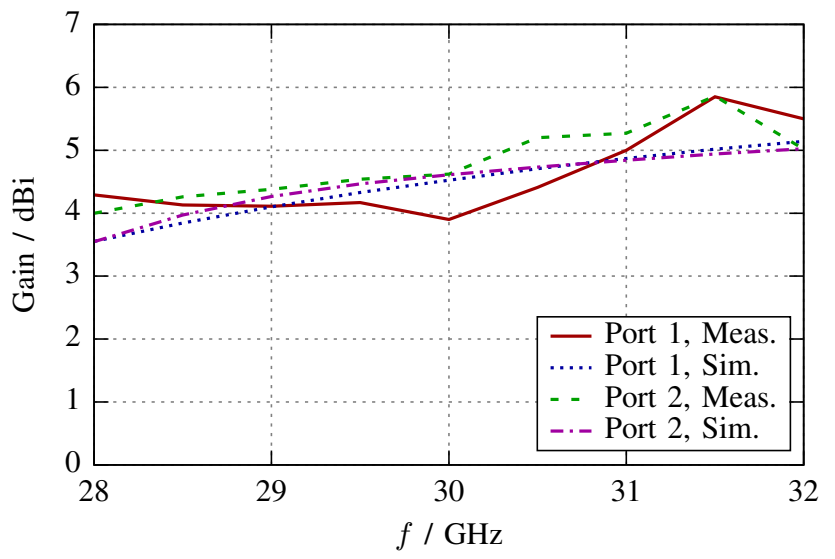

Fig. 19. Measured and simulated gain of the embedded dual-polarized stacked patch antenna.

Despite the asymmetric feeding structure for each polarization, the radiation patterns of the antenna are mainly dominated by the square aperture including the upper patch and the metal grid structure. Apart from minor differences due to technology-based tolerances, this has been likewise confirmed by the experimental results. In the following the scan performance of the proposed antenna within a larger array environment is illustrated by the embedded element patterns of port 1. The results of the central element are shown in Fig. 20 at 29,30 , and $31 \mathrm{GHz}$, respectively.

The measured co-polarized radiation diagrams in the Eand H-plane are very close to the simulations, even for large off-boresight angles. In addition, the element diagram 


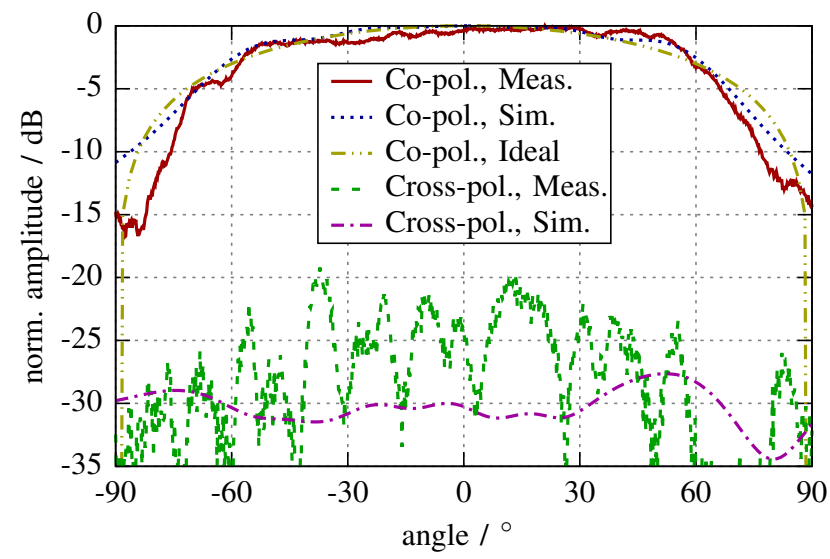

(a) $29 \mathrm{GHz}$, E-plane

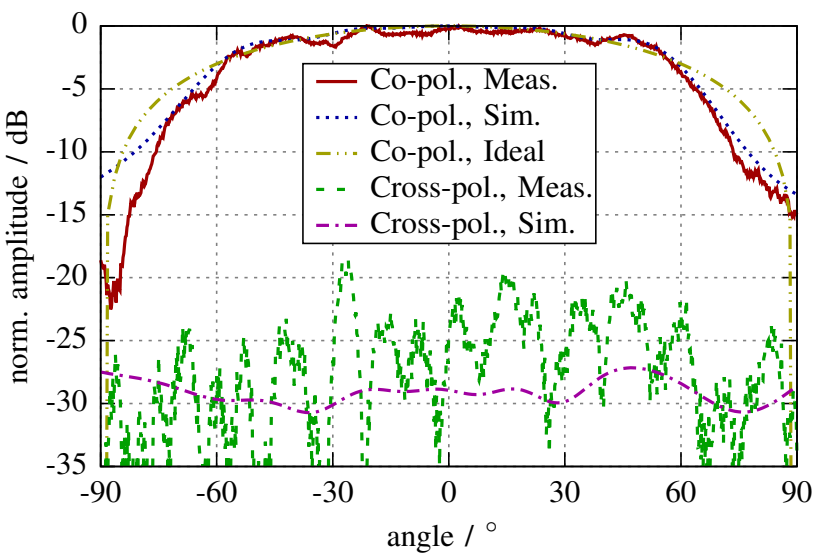

(c) $30 \mathrm{GHz}$, E-plane

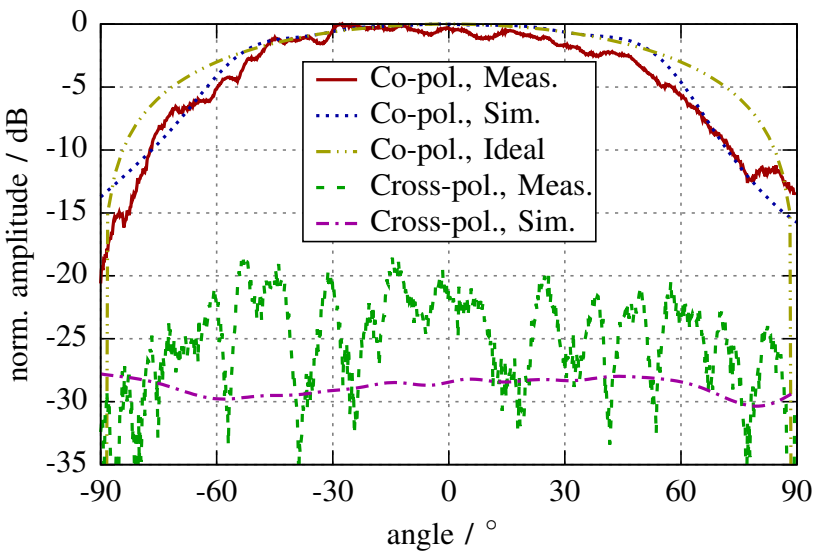

(e) $31 \mathrm{GHz}$, E-plane

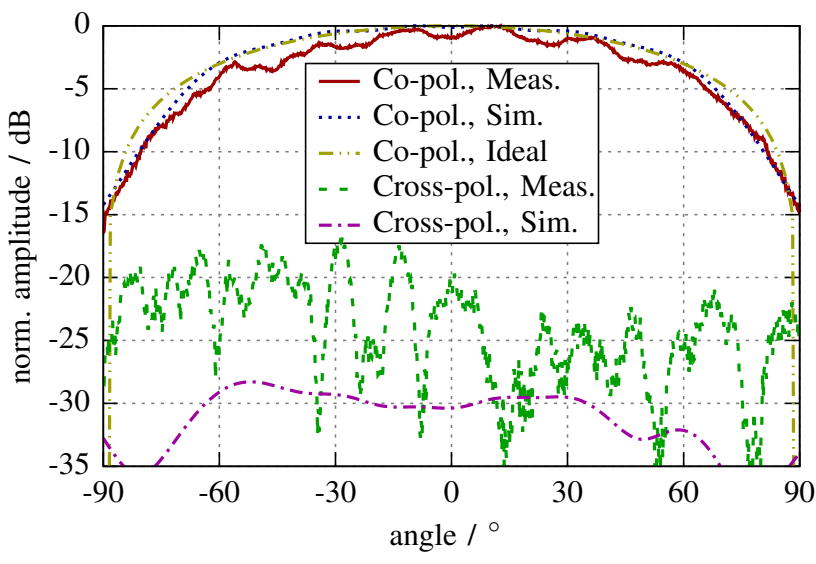

(b) $29 \mathrm{GHz}$, H-plane

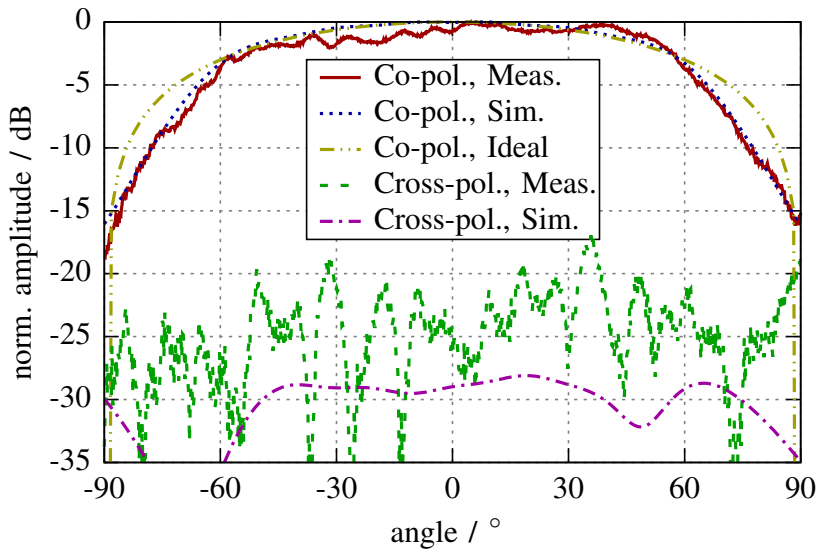

(d) $30 \mathrm{GHz}, \mathrm{H}$-plane

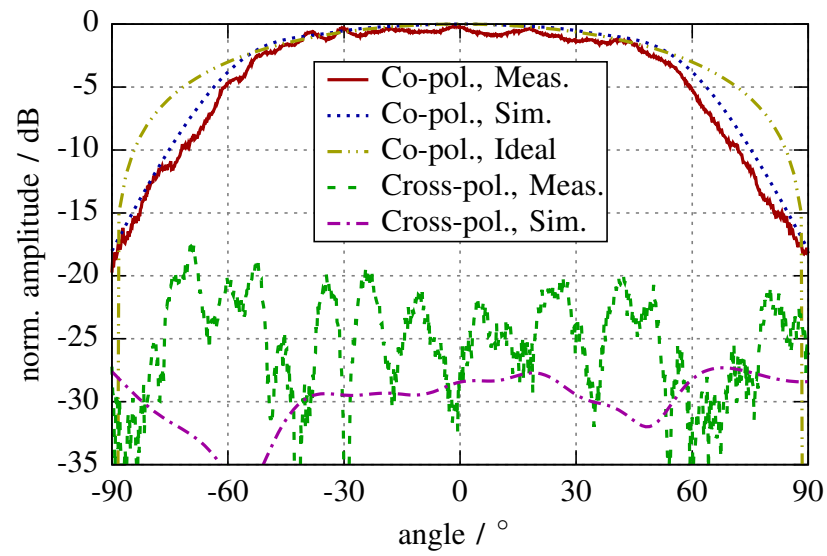

(f) $31 \mathrm{GHz}, \mathrm{H}-$ plane

Fig. 20. Measured and simulated radiation diagrams of the embedded dual-polarized stacked patch antenna (port 1).

performance compared with the ideal $\cos (\vartheta)$ gain degradation indicates the antenna's excellent scan efficiency within the intended scan volume of $\pm 60^{\circ}$. The ripples that are visible to some extent in all measurements originate from parasitic radiation at the edges of finite array. Hence, for larger antenna arrays their impact on the radiation characteristics will degrade gradually. The measured cross-polarization levels for port 1 and 2 are lower than $-15 \mathrm{~dB}$ in both principle planes. Apparently, the measured values are higher as predicted by the full-wave simulations. This can be explained by the conceptional asymmetry which is present only in the realized array configuration, as mentioned above.

\section{CONCLUSION}

A planar hybrid design of a dual-polarized aperture stacked patch antenna for wide angle scanning up to $\pm 60^{\circ}$ in the E- and H-plane has been presented. Parasitic effects of the array antenna degrading the intended scan volume have been elaborated and suppressed efficiently in the operational band. As a critical parameter for integration into a folded 
reflectarray transceiver, the port decoupling between the two polarizations remains high even for larger elevation angles. Transmission coefficients to the individual Floquet modes have been introduced to evaluate its high scanning efficiency. From the technology perspective, the proposed antenna architecture relaxes the overall PCB fabrication process but tends to a more complex assembly in return. Excellent RF performance and reproducibility have been verified by measurements of several antenna prototypes.

\section{ACKNOWLEDGMENT}

The authors would like to thank Markus Laudien of Ansys Germany for the valuable support in providing an optimum simulation setup.

\section{REFERENCES}

[1] E. Feltrin and E. Weller, "New frontiers for the mobile satellite interactive services," in 5th Advanced Satellite Multimedia Systems Conference and the 11th Signal Processing For Space Communications Workshop, 2010, pp. 155-161.

[2] L. Marcellini, R. Lo Forti, and G. Bellaveglia, "Future developments trend for ku and ka antenna for satcom on the move," in Proceedings of the 5th European Conference on Antennas and Propagation, 2011, pp. 2346-2350.

[3] A. Stark, A. Dreher, H. Fischer, A. Geise, R. Gieron, M. Heckler, S. Holzwarth, C. Hunscher, A. Jacob, K. Kuhlmann, O. Litschke, D. Lohmann, W. Simon, F. Wotzel, and D. Zahn, "Santana: Advanced electronically steerable antennas at ka-band," in 3rd European Conference on Antennas and Propagation, Mar. 2009, pp. 471-478.

[4] E. Meniconi, V. Ziegler, R. Sorrentino, and T. Chaloun, "3D integration technologies for a planar dual band active array in ka-band," in European Microwave Conference, Oct. 2013, pp. 215-218.

[5] D. Pilz and W. Menzel, "Folded reflectarray antenna," Electronics Letters, vol. 34, no. 9, p. 832, 1998.

[6] T. Chaloun, W. Menzel, F. Tabarani, T. Purtova, H. Schumacher, M. Kaynak, Q. Luo, S. Gao, R. Starec, and V. Ziegler, "Wide-angle scanning active transmit/receive reflectarray," IET Microwaves, Antennas \& Propagation, vol. 8, no. 11, pp. 811-818, Aug. 2014.

[7] A. Zaghloul, R. Gupta, E. Kohls, L. Sun, and R. M. Allnutt, "Low cost flat antennas for commercial and military satcom terminals," in Military Communications Conference, vol. 2, 2001, pp. 795-799.

[8] S. Vaccaro, F. Tiezzi, D. Llorens, M. F. Ra, and C. D. G. de Oro, "Kuband low profile antennas for mobile satcom," in 4th Advanced Satellite Mobile Systems, 2008, pp. 24-28.

[9] J. Huang and J. Encinar, Reflectarray Antennas, ser. IEEE Press Series on Electromagnetic Wave Theory. John Wiley \& Sons, Inc., 2007.

[10] T.-H. Chio and D. Schaubert, "Parameter study and design of wide-band widescan dual-polarized tapered slot antenna arrays," IEEE Transactions on Antennas and Propagation, vol. 48, no. 6, pp. 879-886, Jun. 2000.

[11] J. Remez, A. Segal, and R. Shansi, "Dual-polarized wideband widescan multibeam antenna system from tapered slotline elements array," IEEE Antennas and Wireless Propagation Letters, vol. 4, pp. 293-296, 2005.

[12] D. Pozar and D. H. Schaubert, "Analysis of an infinite array of rectangular microstrip patches with idealized probe feeds," IEEE Transactions on Antennas and Propagation, vol. 32, no. 10, pp. 1101-1107, 1984.

[13] D. Pozar, "Analysis of an infinite phased array of aperture coupled microstrip patches," IEEE Transactions on Antennas and Propagation, vol. 37, no. 4, pp. 418-425, Apr. 1989.

[14] R. B. Waterhouse, "The use of shorting posts to improve the scanning range of probe-fed microstrip patch phased arrays," IEEE Transactions on Antennas and Propagation, vol. 44, no. 3, pp. 302-309, 1996.

[15] F. Zavosh and J. Aberle, "Infinite phased arrays of cavity-backed patches," IEEE Transactions on Antennas and Propagation, vol. 42, no. 3, pp. 390-398, Mar. 1994.

[16] D. Zhou, C. Zhang, S. Gao, T. Chaloun, W. Menzel, and V. Ziegler, "New multi-layer millimetre-wave folded reflectarray antennas for satellite communications," in 6th European Conference on Antennas and Propagation, 2012, pp. 821-824.
[17] M. Awida, A. Kamel, and A. Fathy, "Analysis and design of wide-scan angle wide-band phased arrays of substrate-integrated cavity-backed patches," IEEE Transactions on Antennas and Propagation, vol. 61, no. 6, pp. 3034-3041, 2013.

[18] R. Waterhouse, "Design and performance of large phased arrays of aperture stacked patches," IEEE Transactions on Antennas and Propagation, vol. 49, no. 2, pp. 292-297, 2001.

[19] A. K. Bhattacharyya, Phased Array Antennas: Floquet Analysis, Synthesis, BFNs and Active Array Systems, ser. Wiley Series in Microwave and Optical Engineering. John Wiley \& Sons, Inc., 2006.

[20] G. Knittel, A. Hessel, and A. Oliner, "Element pattern nulls in phased arrays and their relation to guided waves," Proceedings of the IEEE, vol. 56, no. 11, pp. 1822-1836, 1968.

[21] C. Nehra, A. Hessel, J. Shmoys, and J. Stalzer, H., "Probe-fed stripelement microstrip phased arrays: E- and H-plane scan resonances and broadbanding guidelines," IEEE Transactions on Antennas and Propagation, vol. 43, no. 11, pp. 1270-1280, 1995.

[22] A. Stark and A. Jacob, "A broadband vertical transition for millimeterwave applications," in 38th European Microwave Conference, Oct 2008, pp. 476-479.

[23] HFSS version 14.0.1, Ansys Cooperation, Canonsburg, PA, USA, 2012.

[24] K. Ghorbani and R. Waterhouse, "Dual polarized wide-band aperture stacked patch antennas," IEEE Transactions on Antennas and Propagation, vol. 52, no. 8, pp. 2171-2174, Aug. 2004.

[25] P. Brachat and J. M. Baracco, "Dual-polarization slot-coupled printed antennas fed by stripline," IEEE Transactions on Antennas and Propagation, vol. 43, no. 7, pp. 738-742, 1995.

[26] A. Bhattacharyya, O. Fordham, and Y. Liu, "Analysis of stripline-fed slot-coupled patch antennas with vias for parallel-plate mode suppression," IEEE Transactions on Antennas and Propagation, vol. 46, no. 4, pp. 538-545, Apr. 1998.

[27] S. Targonski, R. Waterhouse, and D. Pozar, "Design of wide-band aperture-stacked patch microstrip antennas," IEEE Transactions on Antennas and Propagation, vol. 2, no. 9, pp. 224-1251, 1998.

[28] C.-C. Liu, A. Hessel, and J. Shmoys, "Performance of probe-fed microstrip-patch element phased arrays," IEEE Transactions on Antennas and Propagation, vol. 36, no. 11, pp. 1501-1509, 1988.

[29] R. E. Collin, Field Theory of Guided Waves, 2nd ed., ser. The IEEE Press Series on Electromagnetic Wave Theory. John Wiley \& Sons, Inc., 1991.

[30] R. E. Collin and F. Zucker, Antenna theory, part 2, ser. Inter-University Electronics Series. McGraw-Hill, 1969, vol. 7.

[31] D. Pozar, "The active element pattern," IEEE Transactions on Antennas and Propagation, vol. 42, no. 8, pp. 1176-1178, Aug. 1994.

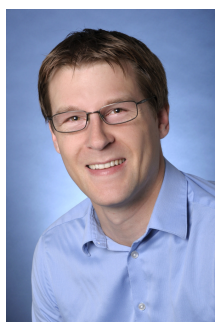

Tobias Chaloun (S'10) received the Dipl.-Ing. degree in electrical engineering from the University of Ulm, Germany, in 2010. Since 2010 he is with the Institute of Microwave Techniques, University of Ulm, and is currently working toward the Ph.D. degree. His current research interests include multilayer antennas and circuits, millimeter-wave packaging and interconnects, phased array antenna systems and millimeter-wave radar sensors. He serves as a reviewer for multiple IEEE TRANSACTIONS and Conferences. Mr. Chaloun was the recipient of the Best Paper Award at the German Microwave Conference in 2015. 


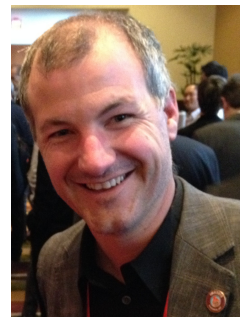

Volker Ziegler (A'01-M'01-SM'09) received his Dipl.-Ing. degree in electrical engineering and his Dr.-Ing. degree (with honors) both from the University of Ulm, Germany, in 1997 and 2001, respectively. From 2002 to 2003, he was member of the "Knowledge Exchange Group for Research and Technology" at the DaimlerChrysler AG in Stuttgart, Germany. During this trainee period, he was working at the University of Michigan, Ann Arbor, USA on the design of GaN high-power MMICs and at United Monolithic Semiconductors, Orsay, France on the modeling and characterisation of III-V semiconductor devices. Afterwards, he joined EADS Innovation Works, Ottobrunn, Germany, where he became an EADS Expert for "Microwave Technologies and Systems" in 2007. From 2013 to 2015, he was the Head of Team RF and Waveforms within AIRBUS Group Innovations. Since 2016, he is the Head of Team Automatic Flight Systems, responsible for the research performed in the field of automated systems including integrated perception, trajectory optimization, image processing and virtual reality for human-machine-interfaces. Volker Ziegler is senior member of the IEEE, member of the IEEE MTT-S Technical Coordinating Committee 21 on RF-MEMS and member of the IEEE MTT Antennas and Propagation German Chapter Executive Board. He served twice as Associated Editor for the International Journal of Microwave and Wireless Technologies and was for several years a member of the Technical Program Committee for the European Microwave Week. He authored or co-authored more than 70 papers, holds eleven patents and is an industrial advisor of the "Component Technical Board on Microwaves" for the European Space Agency.

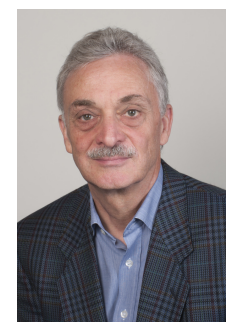

Wolfgang Menzel (M'89-SM'90-F'01) received the Dipl.-Ing. degree in electrical engineering from the Technical University of Aachen, Germany, in 1974, and the Dr-Ing. degree from the University of Duisburg, Germany, in 1977. From 1979 to 1989 , he was with the Millimeter-Wave Department, AEG, Ulm, Germany [now Airbus Defense, and Space], heading the entire Millimeter-Wave Department from 1985 to 1989. In 1989, he became a Full Professor with the Institute of Microwave Techniques, University of Ulm, Germany. His areas of interest are multilayer planar circuits, antennas, millimeter-wave and microwave interconnects and packaging, and millimeter-wave systems. Dr. Menzel is a Fellow of the IEEE. He was an associate editor for the IEEE TRANSACTIONS ON MICROWAVE THEORY AND TECHNIQUES (2003-2005). From 1997 to 1999, he was a Distinguished Microwave Lecturer for Microwave/Millimeter Wave Packaging. From 1997 to 2001, he chaired the German IEEE Microwave Theory and Techniques (MTT)/Antennas and Propagation (AP) Chapter. He is the recipient of the 2002 European Microwave Prize and the 2013 IEEE Microwave Application Award. 